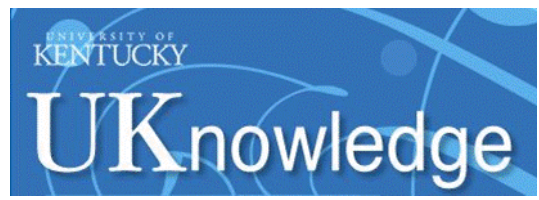

University of Kentucky

UKnowledge

Biosystems and Agricultural Engineering Faculty Publications

\title{
Methods for Calculating Relative Cross-Track Error for ASABE/ISO Standard 12188-2 from Discrete Measurements
}

Joseph D. Rounsaville

University of Kentucky, joeyrounsaville@hotmail.com

Joseph S. Dvorak

University of Kentucky, joe.dvorak@uky.edu

Timothy S. Stombaugh

University of Kentucky, tim.stombaugh@uky.edu

Follow this and additional works at: https://uknowledge.uky.edu/bae_facpub

Part of the Bioresource and Agricultural Engineering Commons

Right click to open a feedback form in a new tab to let us know how this document benefits you.

\section{Repository Citation}

Rounsaville, Joseph D.; Dvorak, Joseph S.; and Stombaugh, Timothy S., "Methods for Calculating Relative Cross-Track Error for ASABE/ISO Standard 12188-2 from Discrete Measurements" (2016). Biosystems and Agricultural Engineering Faculty Publications. 41.

https://uknowledge.uky.edu/bae_facpub/41

This Article is brought to you for free and open access by the Biosystems and Agricultural Engineering at UKnowledge. It has been accepted for inclusion in Biosystems and Agricultural Engineering Faculty Publications by an authorized administrator of UKnowledge. For more information, please contact UKnowledge@lsv.uky.edu. 


\section{Methods for Calculating Relative Cross-Track Error for ASABE/ISO Standard 12188-2 from Discrete Measurements}

Digital Object Identifier (DOI)

https://doi.org/10.13031/trans.59.11902

Notes/Citation Information

Published in Transactions of the ASABE, v. 59, issue 6, p. 1609-1616.

(C) 2016 American Society of Agricultural and Biological Engineers

The copyright holder has granted the permission for posting the article here. 


\title{
METHODS FOR CALCULATING RELATIVE CROSS-TRACK ERROR FOR ASABE/ISO STANDARD 12188-2 FROM DISCRETE MEASUREMENTS
}

\author{
J. Rounsaville, J. Dvorak, T. Stombaugh
}

\begin{abstract}
ASABE/ISO Standard 12188-2 provides test procedures for positioning and guidance systems in agricultural vehicles during straight and level travel. While the standard provides excellent descriptions of test procedures, it does not provide detail on methods to carry out the calculations necessary to calculate relative cross-track error (XTE), which is the primary error statistic used to judge accuracy. Given the travel speed and sampling constraints provided by the standard, the difference between a method based on nearest points or one based on path interpolation could hypothetically be as large as $25 \mathrm{~cm}$. In this project, the standard was used to estimate the guidance accuracy of a relatively low-accuracy vehicle at 1.25 and $0.5 \mathrm{~m} \mathrm{~s}^{-1}$. At $1.25 \mathrm{~m} \mathrm{~s}^{-1}$, a basic nearest point calculation overestimated mean XTE by $0.8 \mathrm{~cm}$, or $8.2 \%$. The location sampling density was much higher with a $0.5 \mathrm{~m} \mathrm{~s}^{-1}$ travel speed, and mean XTE was only overestimated by $0.1 \mathrm{~cm}$ with the nearest point method. There are clearly situations where the calculation method will affect results, and the use of the more complicated methods explained in this article are suggested when using this standard.
\end{abstract}

Keywords. Autonomous, Cross-track error, Guidance, Standard, XTE.

$\mathrm{T}$ he use of automatic vehicle guidance in an agricultural setting has a well-documented history (Heraud and Lange, 2009). While the most common application of autonomous guidance may be the guidance of tractors during field operations, new applications continue to develop. Several recent projects have focused on applications that range from intercropping (Dybro, 2015), to mechanical weeding (Gai, 2015), to disease detection (Dybro, 2015), to vineyard management (Rovira-Mas et al., 2015), to field scouting and sampling (Rains et al., 2015), and even automatic large-scale turf mowing (Chang, 2015). A defined way to quantify the guidance accuracy of autonomous systems is undeniably important, which led to the development of ASABE/ISO Standard 12188-2:2012. As the wide variety of agricultural projects that rely on autonomous guidance continues to expand, this importance can only be expected to increase.

In an agricultural setting, field vehicles are usually driven along a straight line in the field. In this context, any perpendicular deviation from the direction of travel is of upmost importance. Parallel deviations along the travel path merely represent fluctuations in travel speed and are less critical. A

Submitted for review in May 2016 as manuscript number MS 11902; approved for publication by the Machinery Systems Community of ASABE in September 2016.

Publication No. 16-05-103 of the Kentucky Agricultural Experiment Station, published with the approval of the Director.

The authors are Joseph Rounsaville, ASABE Member, Graduate Student, Joseph Dvorak, ASABE Member, Assistant Professor, and Timothy Stombaugh, ASABE Member, Extension Professor, Department of Biosystems and Agricultural Engineering, University of Kentucky, Lexington, Kentucky. Corresponding author: Joseph Rounsaville, 128 C.E. Barnhart Building, University of Kentucky, Lexington, KY 40546-0276; phone: 859-257-3000; e-mail: joseph.rounsaville@uky.edu. standard calculation in capturing the perpendicular deviation and characterizing the accuracy of a guidance system is the cross-track error (Borhaug and Pettersen, 2005), which is calculated as the horizontal deviation from the intended travel path. Cross-track errors can then be used to estimate the overall 2D positional error statistics (Sharp and $\mathrm{Yu}$, 2012) or perform comparisons. However, a cross-track error measurement requires a known reference line that describes the intended path, and this is inconvenient in an agricultural setting. This issue led to the development of the relative cross-track error (XTE), which does not require a known reference line and instead relies on the difference between two passes of a vehicle when programed to drive along the same path. ASABE/ISO Standard 12188-2:2012 is based on relative cross-track error and defines XTE as the "lateral deviation of the Representative Vehicle Point (RVP) from the desired path determined from the previous paths of the RVP when guided along the same test course" (ASABE/ISO, 2012). As the term implies, this is a relative measurement between two travel paths, which means that this differential technique could miss systematic bias errors in the data if they are present. However, this is a necessary concession to make when faced with the inconvenience of a known reference line in a typical agricultural setting.

ASABE/ISO Standard 12188-2:2012 provides test procedures for positioning and guidance systems in agriculture during straight and level travel. The test procedure calls for autonomous travel down and back on a straight test course established using an A-B line. While autonomously traveling along the designated path, precise location measurements of the vehicle are taken at a sampling rate of at least $10 \mathrm{~Hz}$. These measurements are taken at a consistent point on the vehicle, its RVP. The differences between the out- 
bound travel and the return travel are used to define the XTE, which becomes the metric used in reporting the guidance accuracy. The XTE is to be calculated at three different speeds, "slow" $\left(0.1 \mathrm{~m} \mathrm{~s}^{-1}\right)$, "medium" $\left(2.5 \mathrm{~m} \mathrm{~s}^{-1}\right)$, and "fast" $(5.0 \mathrm{~m}$ $\mathrm{s}^{-1}$ ), to capture operating characteristics at the variety of speeds required for different agricultural tasks. It is also to be calculated with different time intervals between the outbound and return paths to determine pass-to-pass and longterm accuracy. The standard provides excellent descriptions of test procedures for evaluating the performance of automated vehicle guidance systems. However, it does not provide detail on methods to carry out the calculations necessary to produce the data that must be reported based on the data collected in the experiment.

At first inspection, this seems uncomplicated. We only need to calculate the lateral deviation of the RVP between the two paths, as the standard states: "The horizontal distance between RVP positions, recorded when traveling in opposite directions, shall represent XTE for every discrete portion of test course segments." The ambiguity in the standard's definition of XTE is that the terms "lateral" and "horizontal" are not defined. The data produced by the test procedure are merely a stream of discrete locations representing the RVP positions in both directions. Horizontal or lateral deviations must be defined based on the fact that they are perpendicular to another line or path. Neither the data nor the standard describe the line or the path from which the lateral deviations will be calculated.

The definition of a line from which to calculate lateral deviations can become complicated. Naturally, the intended travel path cannot be selected, as the impracticality of its use in agricultural settings led to the selection of relative crosstrack error over cross-track error. Possible solutions include a simple nearest point approach, interpolating the travel path between the discrete points and then calculating XTE based on the interpolated paths, or even calculating a line of best fit for use as a reference. The nearest point approach would ignore the "horizontal" requirement and simply base the calculation on the minimum distance between two measurement points on the outbound and return paths. The issue with simple nearest point approaches is that if the location measurements are sparse, then deviations parallel to the path of travel can become more influential than lateral deviations. Path interpolation provides a method to address this by using an interpolation technique to reconstruct the vehicle path and determining XTE based on the minimum deviation between points on the reconstructed paths. A final approach is to produce a reference line based on the data collected, from which the deviations can be decomposed into lateral (perpendicular to) or along (parallel to) the reference line.

A simple illustration reveals the danger of relying on the most basic nearest point method. Using the standard's minimum sample rate of $10 \mathrm{~Hz}$ and the $5 \mathrm{~m} \mathrm{~s}^{-1}$ test speed, the discrete points could be a maximum of $0.5 \mathrm{~m}$ apart. Even if a vehicle perfectly tracked the A-B line on both the outbound and return trips, the individual discrete points in each path could be separated by a maximum of $0.25 \mathrm{~m}$ if the sample locations happened to be perfectly staggered because of timing (fig. 1). Although the vehicle in this example would have

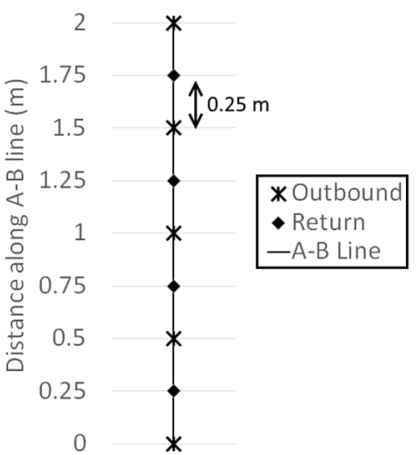

Figure 1. Even though outbound and return paths exactly followed the initial A-B line, the $0.5 \mathrm{~m}$ distance between sample points still permits $0.25 \mathrm{~m}$ between nearest points.

performed perfectly, the distance and the XTE calculated using the nearest point method between any two points on different paths would be $0.25 \mathrm{~m}$.

To illustrate the differences between the methods of calculating XTE, a subset of the ASABE/ISO Standard 121882:2012 test procedure was used on an automated guidance system with relatively low-accuracy travel. The results were then processed using the different methods to illustrate the magnitude of the differences in quantifying the travel of an actual vehicle.

\section{Methods ANd Materials \\ Calculating XTE}

For calculating XTE, the discrete location samples representing the vehicle's path must be represented in a localized Cartesian $(X, Y)$ coordinate system. While some location recording equipment may directly provide this information, others (such as that used in this work) provide output in the form of latitude and longitude coordinates. These were simply transformed using the formula and methods described in ISO Standard 12188-1 (ISO, 2010). This provided inputs to the XTE calculation procedure that consisted of a list of points in Cartesian coordinates for both the outbound and return trips.

\section{Nearest Point (NP) Method}

The NP method is the simplest interpretation of the procedure defined by ISO Standard 12188-2 and consists of determining the distance between a point on the return path and the point closest to it on the outbound path. The simplest way to do this is to calculate the distance to every point on the outbound path from every point on the return path. For each point on the return path, the distances are sorted, and the shortest distance is taken as the XTE for that point. This method effectively interprets the term "lateral" from the ISO Standard 12188-2 definition to be the nearest point on the opposing path. Once appropriate minimum distances are found, the XTE is assigned positive or negative values depending on whether the return path is to the right or left, respectively, of the outbound path.

\section{Linear Path Interpolation (LPI) Method}

The LPI method also requires finding which points are 
closest to each other between the paths. The LPI method applies "lateral" as interpolating between points on the outbound path to allow a minimum perpendicular distance calculation from a point on the return path to the interpolated segment on the outbound path. As with the nearest point method, this process can be performed using brute force. After presenting the algorithm, the necessity of certain steps will be explained with examples. The following algorithm was developed to calculate XTE by interpolating the paths:

1. Heading vector: As the tractor travels along the return path in question, a heading vector can be calculated using the previous point and the current point where:

$R_{2}=\left(X_{C}, Y_{C}\right)$ is the current point on the return path $R_{1}=\left(X_{P}, Y_{P}\right)$ is the previous point on the return path:

$$
\underset{H E A D I N G}{\longrightarrow}=\left(X_{C}-X_{P}\right) \hat{x}+\left(Y_{C}-Y_{P}\right) \hat{y}
$$

2. Find points: In the data, each point on the return path will have a nearest neighbor and a next nearest neighbor on the outbound path. These two points on the outbound path form a line segment where:

$O_{1}=\left(X_{1}, Y_{1}\right)$ is the point on the outbound path closest to $\left(X_{C}, Y_{C}\right)$

$\mathrm{O}_{2}=\left(X_{2}, Y_{2}\right)$ is the neighboring point on the outbound path next closest to $\left(X_{C}, Y_{C}\right)$.

3. Form line: The two points $\left(O_{1}, O_{2}\right)$ along the prior (outbound) path form the following line:

$$
\begin{aligned}
& a x+b y+c=0= \\
& \left(\frac{Y_{2}-Y_{1}}{X_{2}-X_{1}}\right) x-y+\left(Y_{1}-X_{1}\left(\frac{Y_{2}-Y_{1}}{X_{2}-X_{1}}\right)\right)
\end{aligned}
$$

4. Calculate $\left(X_{M}, Y_{M}\right)$ : The minimum perpendicular distance from the above line to $\left(X_{C}, Y_{C}\right)$ will be at point $\left(X_{M}\right.$, $Y_{M}$ ) where:

$$
\begin{aligned}
& X_{M}=\frac{b\left(b X_{C}-a Y_{C}\right)-a c}{a^{2}+b^{2}} \\
& Y_{M}=\frac{a\left(-b X_{C}+a Y_{C}\right)-b c}{a^{2}+b^{2}}
\end{aligned}
$$

5. Check $\left(\boldsymbol{X}_{M}, \boldsymbol{Y}_{M}\right)$ : If point $O_{M}=\left(X_{M}, Y_{M}\right)$ is contained within the line segment $O_{1} O_{2}$, then the point of the relative XTE is $O_{X T E}=\left(X_{X T E}, Y_{X T E}\right)=O_{M}$ and proceed with the algorithm. If $\left(X_{M}, Y_{M}\right)$ lies outside line segment $O_{1} O_{2}$, then eliminate $\mathrm{O}_{2}$ as an option and repeat the algorithm from step 2 to find the next nearest point on the outbound path. If no suitable point $O_{M}$ is found after the desired number of searches, then $\left(X_{X T E}, Y_{X T E}\right)=\left(X_{1}, Y_{1}\right)$ from point $O_{1}$. The Euclidean distance between the segment endpoints can be used to determine when $O_{M}$ is contained within segment $O_{1} O_{2}$ as:

length $\left(O_{1} O_{M}\right)+$ length $\left(O_{M} O_{2}\right) \leq \operatorname{length}\left(O_{1} O_{2}\right)+n \varepsilon$

will be true when $O_{M}$ is contained within the segment for some multiple of machine precision $(n \varepsilon)$.

6. Magnitude of relative XTE: The magnitude of the relative XTE is given as:

$$
|X T E|=\sqrt{\left(X_{X T E}-X_{C}\right)^{2}+\left(Y_{X T E}-Y_{C}\right)^{2}}
$$

7. XTE vector: A vector can be defined from the point of the later path to the point of XTE:

$$
\underset{X T E V E C}{\longrightarrow}=\left(X_{X T E}-X_{C}\right) \hat{x}+\left(Y_{X T E}-Y_{C}\right) \hat{y}
$$

8. Cross product: The cross product between the heading vector and the XTE vector will be purely in the $z$ direction. The sign of this cross product will be the sign of the relative XTE:

$$
(Z) \hat{z}=\operatorname{cross}(\underset{H E A D I N G}{\longrightarrow}, \underset{\text { XTEVEC }}{\longrightarrow})
$$

9. Sign of relative XTE: The sign of $Z$ indicates the sign of the relative XTE. This determines if the return path is to the right or left of the outbound path:

$$
X T E=\operatorname{sign}(Z)^{*}|X T E|
$$

\section{Cubic Path Interpolation (CPI) Method}

The CPI method applies "lateral" to be the same as in the LPI method. However, instead of a linear path interpolation, a cubic interpolation is performed. For this analysis, not-aknot end conditions are assumed. To perform the CPI method, the steps for the LPI method are used, except that step 4 is replaced with distance measured from the cubic curve rather than the line from LPI. A cubic interpolation provides an interpolation method that assumes a smooth travel path for the vehicle, rather than the disjointed travel assumed by linear interpolation.

\section{Perpendicular Component (PC) Method}

The PC method applies "lateral" based on a reference line. The reference line is determined based on a linear regression in the least squares sense on each data set to create a line of best fit (henceforth called $p$ ). The XTE is calculated from the perpendicular (to $p$ ) component of a vector defined by the point on the return path in question $\left(R_{2}\right)$ and the nearest point from the outbound path $\left(O_{1}\right)$. The above algorithm and calculations can now be significantly reduced. The need to find the second nearest point, to interpolate between these points to find the minimum XTE, and to do any endpoint check can be eliminated. This simplified algorithm is given below.

1. $X$ : Create a vector $(X)$ from the current point on the return path in question and the nearest neighbor on the outbound path where:

$R_{2}=\left(X_{C}, Y_{C}\right)$ is the current point on return path

$O_{1}=\left(X_{1}, Y_{1}\right)$ is the point on outbound path closest to $\left(X_{C}\right.$, $\left.Y_{C}\right)$ :

$$
\longrightarrow=\left(X_{C}-X_{1}\right) \hat{x}+\left(Y_{C}-Y_{1}\right) \hat{y}
$$

2. $\boldsymbol{P}$ : Create a vector $(P)$ from the line $p$ using any two points such that this vector points in the same direction as the outbound path.

3. Components: $X$ has a component parallel to $P$ (the projection of $X$ onto $P$ ) and a component perpendicular to $\mathrm{P}$ (the rejection of $X$ from $P$ ): 


$$
\begin{gathered}
\underset{\text { PROJECTION }}{\longrightarrow}=\left(\vec{X} \cdot \frac{\vec{P}}{|\vec{P}|}\right) \frac{\vec{P}}{|\vec{P}|} \\
\underset{\text { REJECTION }}{\longrightarrow}=\frac{\longrightarrow}{\longrightarrow}-\frac{}{\text { PROEJECTIO } \mathrm{N}}
\end{gathered}
$$

4. Cross product: As in the previous algorithm, the cross product between $X$ and $P$ will be purely in the $z$ direction. The sign of this cross product will be the sign of the relative XTE:

$$
(Z) \hat{z}=\operatorname{cross}(\underset{X}{\longrightarrow}, \underset{P}{\longrightarrow})
$$

5. Sign of relative XTE: The sign of $Z$ indicates the sign of the relative XTE. This determines if the return path is to the right or left of the outbound path:

$$
X T E_{P C}=\operatorname{sign}(Z)^{*}|\underset{R E J E C T I O N}{\longrightarrow}|
$$

The examples provided below illustrate the LPI and PC algorithms when used to calculate XTE for point $R_{2}$ (fig. 2). The outbound path is defined by points $\mathrm{O}_{0}, \mathrm{O}_{1}, \mathrm{O}_{2}, \mathrm{O}_{3}$, and the vehicle traveled along that path from $O_{0}$ to $O_{3}$. The later return path is defined by points $R_{0}, R_{1}, R_{2}, R_{3}$, and the vehicle traveled from $R_{0}$ to $R_{3}$. The two points on the prior pass closest to $R_{2}$ are $O_{1}$ and $O_{2}$. Figure 2a illustrates a situation in which point $\left(X_{M}, Y_{M}\right)$ lies within the line segment. Consider point $R_{2}$ of the return path. Points $R_{1}$ and $R_{2}$ form the heading vector, and point $\left(X_{M}, Y_{M}\right)$ falls on the line segment $\mathrm{O}_{1} \mathrm{O}_{2}$. Therefore, $\left(X_{X T E}, Y_{X T E}\right)=\left(X_{M}, Y_{M}\right)$ is used to calculate the relative XTE. Figure $2 \mathrm{~b}$ illustrates a situation in which a steering correction was made on the outbound path. Because of this, point $\left(X_{M}, Y_{M}\right)$, lies outside the line segment $\mathrm{O}_{1} \mathrm{O}_{2}$ and remains outside of any line segment created by the search process. Therefore, from point $O_{1},\left(X_{X T E}, Y_{X T E}\right)=\left(X_{1}\right.$, $\left.Y_{1}\right)$ is used to calculate the XTE. Figure 2c illustrates the PC method. The magnitude of the perpendicular component of $X$ is the magnitude of the XTE.

\section{Guidance ACCURACY TESTING}

The calculation procedures were tested with a simple autonomous ground vehicle at the University of Kentucky campus in Lexington, Kentucky. The vehicle was a custom $15.6 \mathrm{~kW}$ tractor that used electric propulsion drive and electric steering control. Its size and form factor were similar to commercial tractors of a comparable power rating. Vehicle control was provided by a Pixhawk autopilot, which is often used in unmanned aerial systems (UAS). When used with a UAS, the Pixhawk almost always uses uncorrected GNSS for guidance. It is capable of handling standard NEMA-formatted inputs from external GNSS systems, so for this vehicle, a Trimble 5800, operating in real-time kinematic (RTK) mode and providing location samples at $10 \mathrm{~Hz}$, was used for position feedback. The guidance system was tuned following standard procedures for the Pixhawk when used to control ground vehicles (ArduPilot, 2016). Although the system received position information from an RTK system, the navigation algorithms were primarily designed for UAS, so the guidance accuracy did not approach that achieved by the advanced control systems on modern tractors.

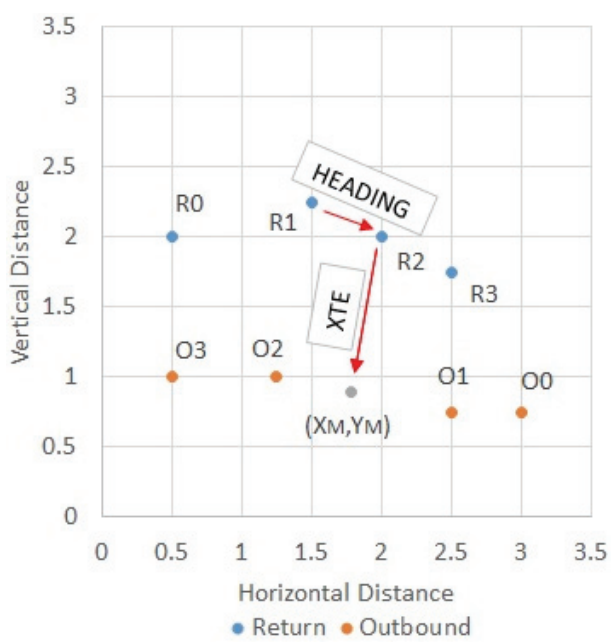

(a)

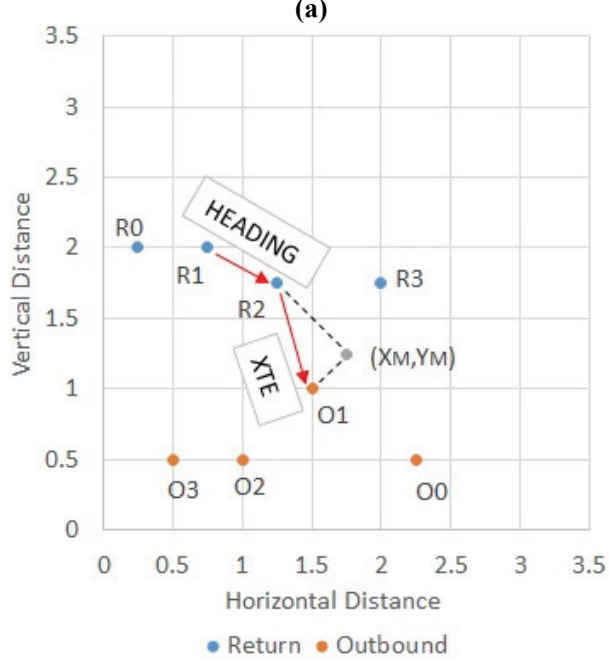

(b)

Figure 2. XTE calculation example using the LPI method.

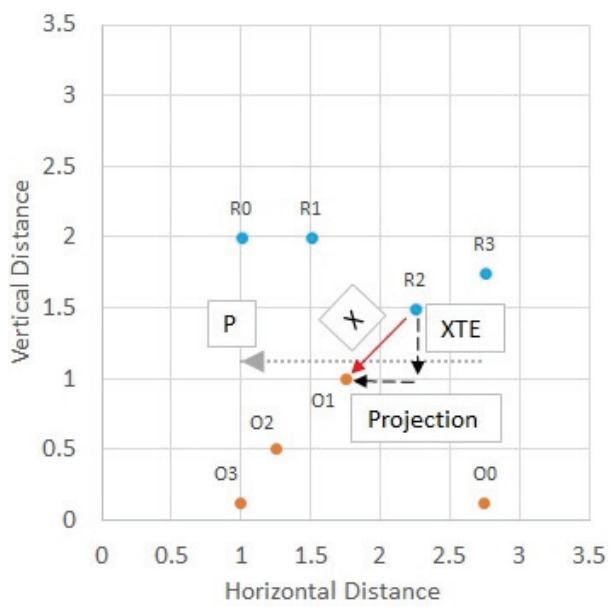

(c)

Figure 2(c). XTE calculation example using the PC method.

To provide insight on the guidance accuracies that could be expected using such a simple system, a subset of the ASABE/ISO Standard 12188-2:2012 test procedures was performed. This testing only considered two travel speeds and immediate revisit times. Location information was only based on the single RTK system mounted on the vehicle and 
operating at $10 \mathrm{~Hz}$ but was recorded separately from the navigation system. Although this does not represent the equipment and accuracy levels commonly used with this standard in testing high-precision vehicles, it created streams of location data representing outbound and return paths that satisfied the frequency requirements of the standard. These streams of data representing the paths were then processed to provide a basic idea of the relative XTE for this system. However, in processing the data from the experiment, it became apparent that there could be issues with the interpretation of the location data, which led to the development of the methods presented here.

For the purposes of this project, only the outbound and immediate return paths used for calculating pass-to-pass XTE were recorded. The experiment was conducted at speeds of 0.5 and $1.25 \mathrm{~m} \mathrm{~s}^{-1}$, which were the minimum speed supported by the autopilot and the maximum speed allowed for safety in this unmanned system. These speeds were set within the autopilot, which then maintained the travel speed at the desired level for that test.

A paved lot was chosen that provided the minimum length of $100 \mathrm{~m}$ in accordance with ASABE/ISO Standard 12188-2. The A-B line was measured beforehand, and desired waypoints were marked in accordance with ASABE/ISO Standard 12188-2. The vehicle was driven along the path under automatic control. Manual control was assumed to turn the vehicle around, and then automatic control was re-initiated for the return trip along the path.

\section{RESULTS}

The simple Pixhawk autopilot was not designed for highly accurate ground travel, so some weaving was noticeable in the travel paths recorded during the test. Figure 3 represents the travel paths based on lateral deviation from $p$, which have been rotated to be parallel with the $x$-axis. These plots show the vehicle position in relation to travel along the return path. Therefore, the vehicle began the outbound path at the $100 \mathrm{~m}$ mark in the plot and traveled until reaching the $0 \mathrm{~m}$ mark. It then turned around and traveled back on the return path, starting at $0 \mathrm{~m}$ and progressing to $100 \mathrm{~m}$. Noticeable in the plots is lower-frequency weaving and higher-frequency steering corrections present in both test runs. In the run at $0.5 \mathrm{~m} \mathrm{~s}^{-1}$, the low-frequency weaving has a period of approximately $10 \mathrm{~m}$,

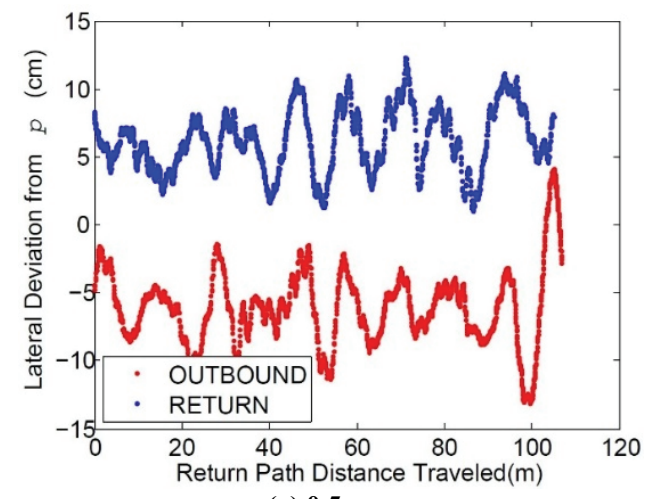

(a) $0.5 \mathrm{~m} \mathrm{~s}$ while at a travel speed of $1.25 \mathrm{~m} \mathrm{~s}^{-1}$ the period is between 20 to $30 \mathrm{~m}$. The higher-frequency corrections are most noticeable when they occurred at a peak or valley of the lower-frequency oscillations, but they can be seen at other locations as well. Although all tests (outbound and return and at both speeds) used the same guidance line, the vehicle constantly traveled slightly to the left of the line, generating an offset between the outbound and return paths in both tests. The large deviation at approximately $100 \mathrm{~m}$ captures the vehicle settling on the A-B line to begin the outbound path. Finally, the location sampling was constant at $10 \mathrm{~Hz}$, so at the slower travel speed there is a noticeably greater density of sampling points. Overall, these characteristics created a complicated but realistic set of paths with which to calculate XTE.

When comparing the mean XTE calculated using each method (table 1), it is apparent that the NP method performed very differently from the other three methods, and that PC, CPI, and LPI perform nearly identically. Travel speed and the resulting difference in density of location measurements affected the differences between NP and the other methods. At the $1.25 \mathrm{~m} \mathrm{~s}^{-1}$ travel rate, mean XTENP was $0.8 \mathrm{~cm}(8.2 \%)$ larger than the other mean XTE values, but the difference was only $0.1 \mathrm{~cm}$ at the $0.5 \mathrm{~m} \mathrm{~s}^{-1}$ travel speed. As a comparison, assuming perfect navigation but staggered position sampling points, as shown in figure 1, the differences would be 6.25 and $2.5 \mathrm{~cm}$ for travel at 1.25 and $0.5 \mathrm{~m} \mathrm{~s}^{-1}$, respectively. The differences observed in the actual experiment are nowhere near these maximum levels; however, this experiment demonstrates that the theoretical weakness of the NP method with sparse location measurements can manifest itself in actual experiments.

Plotting the instantaneous XTE as the vehicle traveled along the path (fig. 4) shows the variability that exists in the XTE value that is hidden when looking at the single mean value. The weaving that was apparent in the original paths (fig. 3) is also noticeable in the plots of XTE calculated using the PC method (fig. 4). At both travel speeds, the XTE was always less than $25 \mathrm{~cm}$, but the XTE was constantly changing

Table 1. Comparison of mean XTE calculated using each method.

\begin{tabular}{ccc}
\hline & \multicolumn{2}{c}{ Mean XTE (cm) } \\
\cline { 2 - 3 } Calculation Technique & At $1.25 \mathrm{~m} \mathrm{~s}^{-1}$ & At $0.5 \mathrm{~m} \mathrm{~s}^{-1}$ \\
\hline Perpendicular component (PC) & -9.81 & -12.33 \\
Cubic path interpolation (CPI) & -9.81 & -12.34 \\
Linear path interpolation (LPI) & -9.81 & -12.34 \\
Nearest point (NP) & -10.61 & -12.44 \\
\hline
\end{tabular}

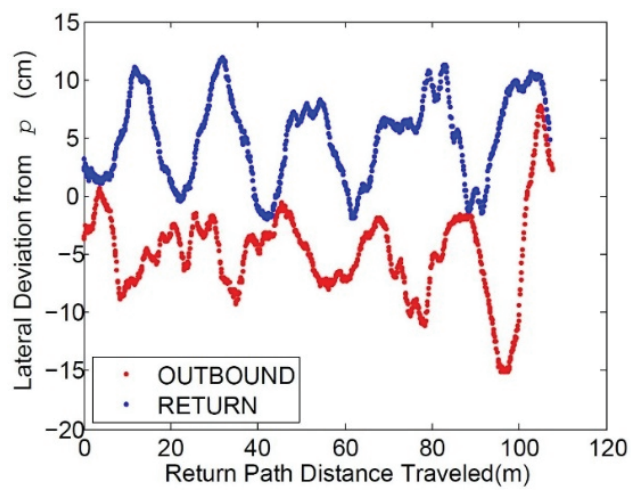

(b) $1.25 \mathrm{~m} \mathrm{~s}^{-1}$

Figure 3. Outbound and return paths for a vehicle traveling at (a) $0.5 \mathrm{~m} \mathrm{~s}^{-1}$ and (b) $1.25 \mathrm{~m} \mathrm{~s}^{-1}$ illustrating the constant weaving and direction changes experienced in this test. 


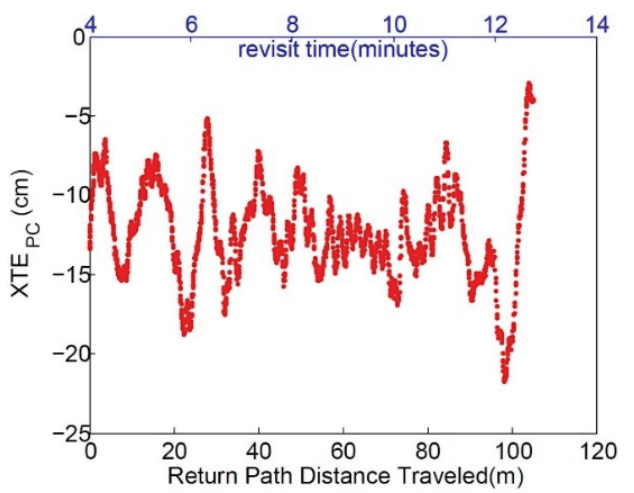

(a) $0.5 \mathrm{~m} \mathrm{~s}$

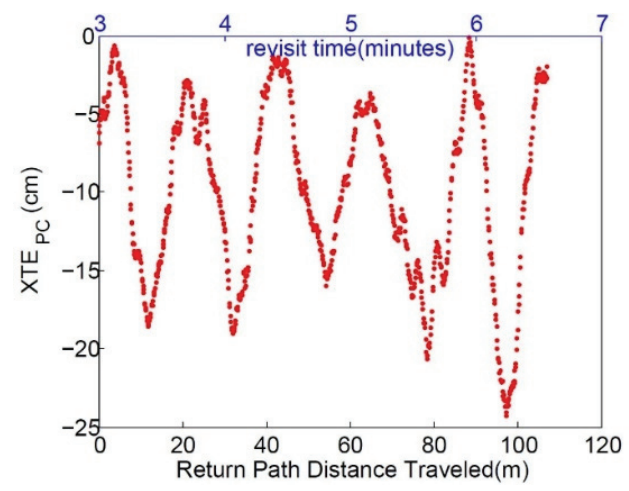

(b) $1.25 \mathrm{~m} \mathrm{~s}^{-1}$

Figure 4. Relative XTE calculated using the PC method for a vehicle traveling at (a) $0.5 \mathrm{~m} \mathrm{~s}^{-1}$ and (b) $1.25 \mathrm{~m} \mathrm{~s}^{-1}$. Only PC is shown because LPI, $\mathrm{CPI}$, and PC were nearly identical.

during vehicle travel.

Just as point XTE varies along the travel path, the difference between the calculation methods can also vary. Figure 5 shows the difference in magnitudes between XTE $E_{N P}$ and $\mathrm{XTE}_{\mathrm{PC}}$ in each of the guidance experiments. The magnitude of $\mathrm{XTE}_{\mathrm{NP}}$ was always greater than or equal to the magnitude of XTE $\mathrm{PC}_{\mathrm{PC}}$. As expected, the lower sampling density at the higher speed permitted higher differences between calculation methods. The maximum differences were almost $5 \mathrm{~cm}$ in the test at $1.25 \mathrm{~m} \mathrm{~s}^{-1}$ but always less than $0.8 \mathrm{~cm}$ at $0.5 \mathrm{~m} \mathrm{~s}^{-1}$. At both speeds, there were certain locations that displayed much higher differences than other locations. At

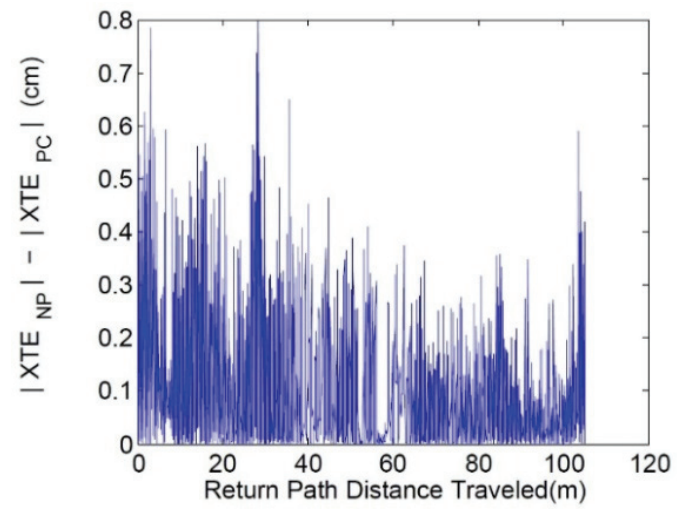

(a) $0.5 \mathrm{~m} \mathrm{~s}$ some locations, these differences remained consistent for five or more meters (e.g., peak at $45 \mathrm{~m}$ in the $1.25 \mathrm{~m} \mathrm{~s}^{-1}$ test). At other locations with increased differences, some points had increased differences, while other nearby points had almost no difference (e.g., peak at $20 \mathrm{~m}$ in the $1.25 \mathrm{~m} \mathrm{~s}^{-1}$ test). Finally, while the average difference between $\mathrm{XTE}_{\mathrm{NP}}$ and XTE $P$ PC was only $0.11 \mathrm{~cm}$ at $0.5 \mathrm{~m} \mathrm{~s}^{-1}$, figure $5 \mathrm{a}$ shows that the point differences were often much larger and that these methods were not as equivalent at this speed as it would seem by only comparing average values.

In contrast to the NP-PC comparison of point XTE values, there was very little difference between the LPI and CPI

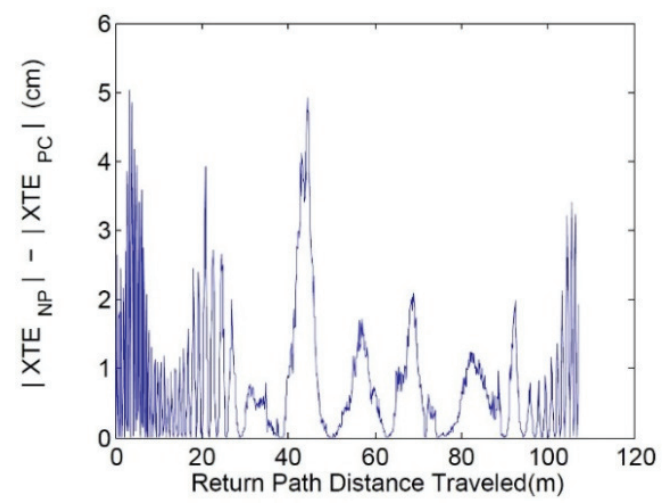

(b) $1.25 \mathrm{~m} \mathrm{~s}^{-1}$

Figure 5. Difference in magnitudes between relative XTE calculated using the PC and NP methods for tests at (a) $0.5 \mathrm{~m} \mathrm{~s}^{-1}$ and (b) $1.25 \mathrm{~m} \mathrm{~s}^{-1}$. The perpendicular component always resulted in a reduction (i.e., improvement) in XTE, so the values are all positive.

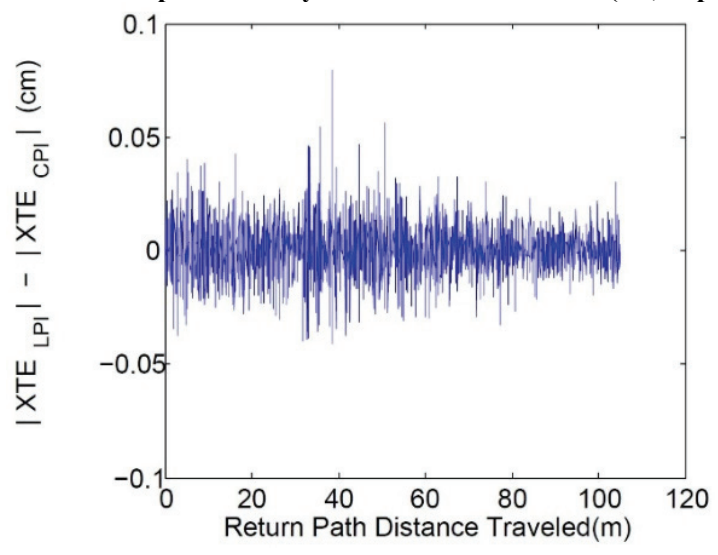

(a) $0.5 \mathrm{~m} \mathrm{~s}$

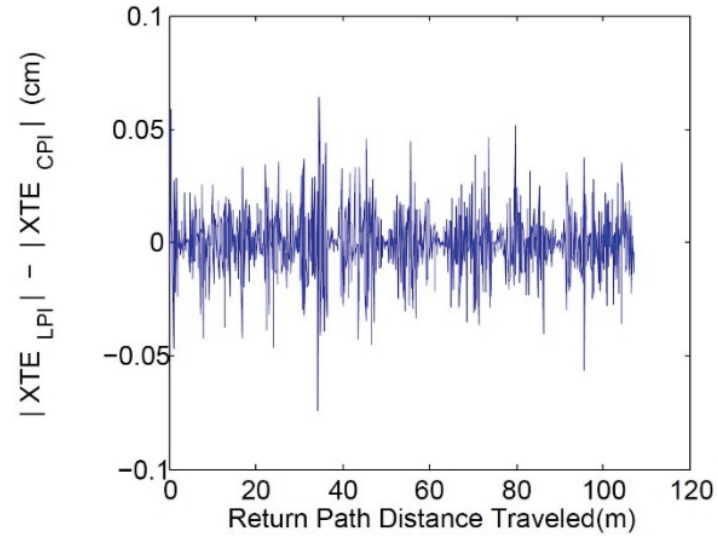

(b) $1.25 \mathrm{~m} \mathrm{~s}^{-1}$

Figure 6. Difference in magnitudes between relative XTE calculated using the LPI and CPI methods for tests at (a) $0.5 \mathrm{~m} \mathrm{~s}^{-1}$ and (b) $1.25 \mathrm{~m} \mathrm{~s}^{-1}$. 


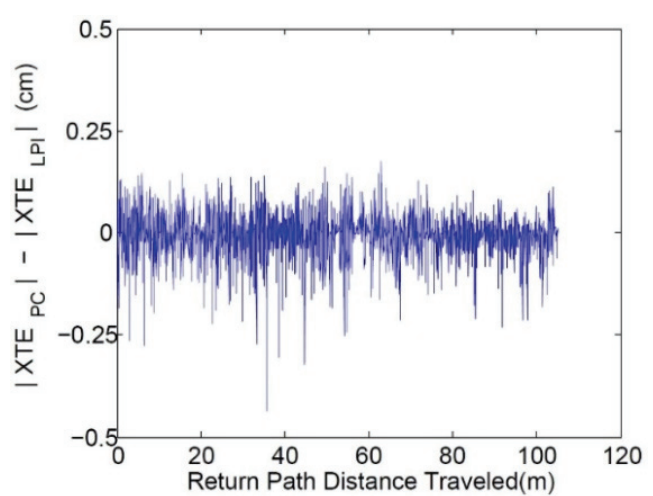

(a) $0.5 \mathrm{~m} \mathrm{~s}^{-1}$

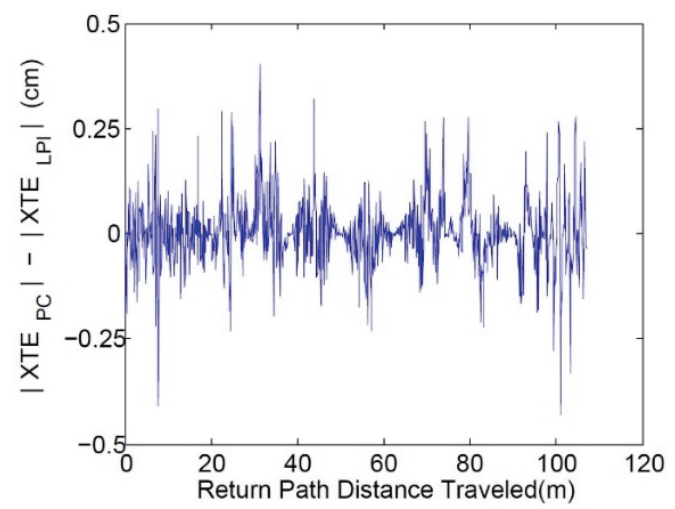

(b) $1.25 \mathrm{~m} \mathrm{~s}^{-1}$

Figure 7. Difference in magnitudes between relative XTE calculated using the LPI and PC methods for tests at (a) $0.5 \mathrm{~m} \mathrm{~s}^{-1}$ and (b) $1.25 \mathrm{~m} \mathrm{~s}^{-1}$.

methods (fig. 6). The magnitude of cubic path interpolation $\mathrm{XTE}\left(\mathrm{XTE}_{\mathrm{CPI}}\right)$ was very comparable to the magnitude of linear path interpolation XTE (XTE $\mathrm{LPI}_{\mathrm{LI}}$. The differences were all less than $1 \mathrm{~mm}$, and they were distributed above and below zero, indicating little systematic bias. Finally, the differences were spread out throughout the length of the path and were not concentrated in certain locations, as was seen in the differences between NP and PC.

The differences between $\mathrm{XTE}_{\mathrm{LPI}}$ and $\mathrm{XTE}_{\mathrm{PC}}$ (fig. 7) were greater than the difference between the two interpolation methods (fig. 6), but the differences were always less than $0.5 \mathrm{~cm}$ and generally distributed around zero. The differences were evenly distributed in the test at $0.5 \mathrm{~m} \mathrm{~s}^{-1}$, but there was a slight clustering of errors in the test at $1.25 \mathrm{~m} \mathrm{~s}^{-1}$. However, this clustering of differences was much less dramatic that that seen in the comparison between NP and PC.

\section{DisCUSSION}

The most apparent result from this work is that the NP method is significantly different from the other methods and appears to overestimate XTE. This difference was illustrated in both the mean XTE values and the point XTE values when they were compared between methods. While XTE $\mathrm{LPI}_{\text {, }}$, $\mathrm{XTE}_{\mathrm{CPI}}$, and XTE $\mathrm{PC}_{\mathrm{PC}}$ had small differences between them, which were all distributed around zero, $X T E_{\mathrm{NP}}$ was always higher than XTEPC, and these differences were clearly clustered around certain points in the travel path.

Using LPI, CPI, or PC required assumptions about either vehicle travel or an appropriate reference line, but all three methods provided similar results in this testing. Several considerations must be made in determining the most appropriate interpolation method to use. Cubic interpolation provides a smooth connection between all the sample points. With low-acceleration travel, cubic interpolation would be highly appropriate. However, if the travel dynamics include sudden shifts in direction, the cubic interpolation requirement for smooth transitions can result in an interpolated path that extends laterally well beyond the actual travel paths, which could introduce errors in the XTE calculation. On the other hand, linear interpolation assumes straight-line travel between sample points with sudden direction changes at each sample point. This may not capture actual vehicle dynamics, and it cannot interpolate a path that extends laterally beyond the sampled location points. Tractor travel dynamics when performing tillage tasks such as plowing are generally slow and would match the low-acceleration assumption for cubic interpolation. Other tasks, such as high-speed planting and tractor application of liquid or solid inputs, can occur at speeds above $16 \mathrm{k} \mathrm{h}^{-1}$. They can also be performed in no-till ground or in growing crops, where the soil surface is rough, which might not produce the slow dynamics assumed by cubic interpolation. The standard permits testing on "agricultural surfaces" like these. Further, the scope of the standard extends beyond tractors to agricultural ground vehicles in general, so high-speed self-propelled sprayers and even future autonomous equipment that might have travel dynamics very different from those of a plowing tractor should be considered. Because of the wide variety of vehicle dynamics that could be encountered on agricultural vehicles, it is difficult to select one interpolation method over another.

The PC method does not require a specific assumption of vehicle travel dynamics, but it does require a travel reference line from which "lateral" is defined. The application of a relative calculation means that this reference line should be set on the data collected in the experiment. Given this limitation, the most appropriate method to determine a travel reference line is from the best-fit line of the data collected. The standard requires straight-line travel during data collection periods, and using a best-fit line ensures that the entire set of collected data is used in determining the travel path.

One method to limit the differences and improve the accuracy of the simple NP calculation is to ensure that the location measurements are taken at high frequency to generate a dense set of points to describe the vehicle paths. In this testing, this was demonstrated by the much lower mean difference $(0.11 \mathrm{~cm})$ between calculation methods when using the more densely sampled paths taken at $0.5 \mathrm{~m} \mathrm{~s}^{-1}$. It is also possible that the most advanced navigation controls in modern field tractors would exhibit less weaving and produce simpler paths, which might result in smaller differences.

The autopilot for the autonomous vehicle used in this testing was not nearly as refined as those in modern field tractors. However, tractors are not the only agricultural vehicles that are expected to use autonomous navigation. All of the previously cited autonomous agricultural vehicles have unique accuracy levels to achieve success in their applications and will be considerably different from the accuracy of a general- 
purpose tractor. Some applications, such as intra-row weeding, require the highest possible accuracy, while others, such as automated pre-plant soil sampling, require much lower levels of navigational accuracy. Because of the expected expansion in autonomously guided agricultural vehicles, it is imperative that the standards used for ascertaining accuracy be welldefined, as they could see much wider application than simply on general-purpose tractors. This work demonstrates that a path interpolation or vector decomposition technique, rather than a simple nearest point method, should be used to determine XTE for documenting navigational accuracy.

\section{Conclusions}

We have presented four different possible methods that could be used to calculate XTE from the raw local data that are produced when following the procedures outlined in ASABE/ISO Standard 12188-2. Three of the methods (LPI, $\mathrm{CPI}$, and PC) produced very similar results, while the NP method provided results that were clearly different. The NP method's strength is the ease with which it can be applied. However, it appears unacceptable for calculating XTE because of its potential to overestimate XTE given the sample rates and speeds required by the standard. Path interpolation, as represented by LPI and CPI, addresses the low density of location measurements by interpolating the vehicle path. The drawbacks of path interpolation are the increased complexity of the calculations and ensuring that the selected interpolation method appropriately reflects the travel of the vehicle in the field. Finally, the PC method produced results very similar to the path interpolation methods, but it required assuming a reference line from the data. This method is relatively simple to implement, but it requires accepting the line of best fit for the travel paths as an appropriate reference from which to determine lateral deviations.

Based on the results of the experiments conducted in this project, there is very little reason to suggest the LPI, CPI, or PC method over the other two methods, as all three methods were reasonable. However, the PC method was simpler to implement in code than the other methods and does not require assumptions on the steadiness, or lack thereof, of agricultural equipment paths. The only additional assumption required of the PC method is that the best-fit line is an acceptable reference line, which would appear to be a perfectly reasonable assumption. Based on the simplicity of the PC method and the fact that it varies very little from any path interpolation technique, it is our suggested method to use when calculating XTE.

\section{ACKNOWLEDGEMENTS}

This work is supported by the USDA National Institute of Food and Agriculture (NIFA) Hatch Multistate project under 1001110 .

\section{REFERENCES}

ArduPilot. (2016). Tuning steering and navigation for a Rover. Retrieved from http://ardupilot.org/rover/docs/tuning-steeringand-navigation-for-a-rover.html
ASABE/ISO. (2012). 12188-2: Tractors and machinery for agriculture and forestry-Test procedures for positioning and guidance systems in agriculture-Part 2: Testing of satellitebased auto-guidance systems during straight and level travel. St. Joseph, MI: ASABE.

Borhaug, E., \& Pettersen, K. (2005). Cross-track control for underactuated autonomous vehicles. In Proc. 44th IEEE Conf. on Decision and Control, and the European Control Conf. (pp. 602-608). Piscataway, NJ: IEEE.

Chang, C.-L., Yang, C., \& Song, G.-B. (2015). Integration of laser scanner and odometry for autonomous robotics lawn-mower. ASABE Paper No. 152190609. St. Joseph, MI: ASABE.

Dybro, N. (2015). Agronomy-based crop production system. ASABE Paper No. 152181293. St. Joseph, MI: ASABE.

Gai, J., Tang, L., \& Steward, B. (2015). Plant recognition through the fusion of $2 \mathrm{D}$ and $3 \mathrm{D}$ images for robotic weeding. ASABE Paper No. 152181371. St. Joseph, MI: ASABE.

Heraud, J. A., \& Lange, A. F. (2009). Agricultural automatic vehicle guidance from horses to GPS: How we got here, and where we are going. ASABE Distinguished Lecture Series No. 33. St. Joseph, MI: ASABE.

ISO. (2010). 12188-1: Tractors and machinery for agriculture and forestry-Test procedures for positioning and guidance systems in agriculture-Part 1: Dynamic testing of satellitebased positioning devices. Geneva, Switzerland: ISO.

Rains, G. C. P., Bazemore, B. W., Ahlin, K., Hu, A.-P., Sadegh, N., \& McMurray, G. (2015). Steps toward an autonomous field scout and sampling system. ASABE Paper No. 152190771. St. Joseph, MI: ASABE.

Rovira-Mas, F. M. R., Millot, C., \& Saiz-Rubio, V. (2015). Navigation strategies for a vineyard robot. ASABE Paper No. 152189750. St. Joseph, MI: ASABE.

Sharp, I., \& Yu, K. (2012). Positional accuracy measurement and error modeling for mobile tracking. IEEE Trans. Mobile Comput., 11(6), 1021-1032.

http://dx.doi.org/10.1109/TMC.2011.119

\section{NOMENCLATURE}

A-B line = imaginary line passing through two points (A and B)

$\mathrm{CPI}=$ cubic path interpolation method

GNSS = global navigation satellite system

LPI = linear path interpolation method

NEMA = National Marine Electronics Association

$\mathrm{NP}=$ nearest point method

$O_{1}=\left(X_{1}, Y_{1}\right)=$ point on outbound path closest to $\left(X_{C}, Y_{C}\right)$

$O_{2}=\left(X_{2}, Y_{2}\right)=$ neighboring point to $O_{1}$ on the outbound path next closest to $\left(X_{C}, Y_{C}\right)$

$O_{M}=\left(X_{M}, Y_{M}\right)=$ minimum perpendicular distance from formed line to $\left(X_{C}, Y_{C}\right)$

$O_{X T E}=\left(X_{X T E}, Y_{X T E}\right)=$ point of the relative XTE

$p=$ line of best fit in the linear regression least squares sense

$\mathrm{PC}=$ perpendicular component method

$R_{1}=\left(X_{P}, Y_{P}\right)=$ previous point on return path in question

$R_{2}=\left(X_{C}, Y_{C}\right)=$ current point on return path in question

$\mathrm{RTK}=$ real-time kinematic

$\mathrm{RVP}=$ representative vehicle point

$\mathrm{UAS}=$ unmanned aerial system

$\mathrm{XTE}=$ relative cross-track error

$\mathrm{XTE}_{\mathrm{NP}}=\mathrm{XTE}$ as calculated by the nearest point method

$\mathrm{XTE}_{\mathrm{LPI}}=\mathrm{XTE}$ as calculated by the linear path interpolation method

$\mathrm{XTE}_{\mathrm{CPI}}=\mathrm{XTE}$ as calculated by the cubic path interpolation method

$\mathrm{XTE}_{\mathrm{PC}}=\mathrm{XTE}$ as calculated by the perpendicular component method 